Cahiers $d u$ MONDE RUSSE

\section{Cahiers du monde russe}

Russie - Empire russe - Union soviétique et États indépendants

45/3-4 | 2004

Varia

\title{
Paul R. Gregory, Valery Lazarev, eds., The economics of forced labor
}

\section{Marta Craveri}

\section{OpenEdition \\ Journals}

Édition électronique

URL : https://journals.openedition.org/monderusse/4194

DOI : 10.4000/monderusse.4194

ISSN : $1777-5388$

Éditeur

Éditions de l'EHESS

\section{Édition imprimée}

Date de publication : 1 juillet 2004

Pagination : 679-681

ISBN : 2-7132-2009-2

ISSN : $1252-6576$

Référence électronique

Marta Craveri, «Paul R. Gregory, Valery Lazarev, eds., The economics of forced labor », Cahiers du monde russe [En ligne], 45/3-4 | 2004, mis en ligne le 06 juillet 2009, consulté le 03 septembre 2022 URL : http://journals.openedition.org/monderusse/4194; DOI : https://doi.org/10.4000/monderusse. 4194

Ce document a été généré automatiquement le 3 septembre 2022.

Tous droits réservés 


\title{
Paul R. Gregory, Valery Lazarev, eds., The economics of forced labor
}

\author{
Marta Craveri
}

\section{RÉFÉRENCE}

Paul R. GREGORY, Valery LAZAREV, eds., The economics of forced labor. The Soviet

Gulag. Stanford, California, Hoover Institution Press, 2003, 212 p.

1 Cet ouvrage, dirigé par Paul Gregory et Valery Lazarev, permet enfin de combler une importante lacune historiographique concernant le rôle du travail forcé dans l'économie soviétique. Le livre, composé d'une préface de Robert Conquest, d'une introduction de P. Gregory, de huit chapitres écrits par différents auteurs et d'une conclusion rédigée par V. Lazarev, a pour but d'individualiser les logiques - politiques ou économiques - qui ont amené à la formation du Goulag, au réseau des camps et des colonies de travail. Certains des chapitres abordent des aspects plus généraux comme les rapports entre le travail forcé et l'industrie ou bien le rôle de l'OGPU/NKVD/MVD dans l'économie soviétique, d'autres sont des études de cas sur certains des camps et des chantiers les plus importants de l'Archipel du Goulag.

2 L'introduction de Paul Gregory fait le point sur les principales connaissances acquises dans l'histoire du Goulag depuis l'ouverture des archives soviétiques: structure administrative, nombre de prisonniers, géographie du réseau des camps et rôle économique.

Dans le premier chapitre, «Forced labor in Soviet industry: the end of the 1930s to the mid-1950s: an overview ", Andrei Sokolov démontre que l'alternance de primes et de mesures draconiennes envers la main-d'œuvre ouvrière a été une constante de la politique industrielle pendant le stalinisme. Par une étude systématique et chronologique de ce système, qu'il appelle politique de la carotte ou du bâton, l'auteur décrit comment, même dans les moments les plus répressifs, le leadership stalinien va combiner coercition et motivation, punition et encouragement, en finissant par aboutir 
à un système contradictoire, inefficace du point de vue économique, improductif et qui sera rapidement abandonné après la mort de Stalin.

4 « The economy of the OGPU, NKVD and MVD of the URSS, 1930-1953: The scale, structure and trends of development » (Oleg Khlevnyuk) reconstruit le rôle économique que la police politique et le commissariat à l'Intérieur vont acquérir tout au long de la période stalinienne en montrant comment les logiques qui sont à la base de la création du Goulag sont initialement et principalement des logiques politiques et ensuite seulement des logiques économiques. Ce sont la répression et la terreur comme instruments de gouvernement du pays qui engendreront la nécessité d'administrer une gigantesque et toujours croissante population concentrationnaire. Celle-ci va progressivement transformer le Goulag en un des acteurs économiques principaux en obligeant ainsi ses responsables à devoir élaborer des stratégies économiques pour respecter les plans qui leur étaient assignés. Khlevnyuk insiste d'une part sur les coûts sociaux et économiques du Goulag et d'autre part sur la suprématie du politique sur l'économique, et prend comme exemple significatif la Grande Terreur, dont le but principal était l'élimination physique des ennemis plutôt que leur utilisation économique. C'est ainsi qu'environ 700000 personnes (en majorité en bonne condition physique et constituant une maind'œuvre hautement spécialisée) furent exécutées sans tenir compte des impératifs de production du Goulag.

5 Un autre exemple de cette argumentation est constitué par l'article de David Nordlander, «Magadan and the economic history of Dalstroi in the 1930s ", qui montre bien que le développement économique du Dalstroi, initié avant 1937 sous la direction de Berzin, fut brutalement interrompu par les répressions de masse qui désorganisèrent complètement le fonctionnement du camp. Par la suite, les tentatives du nouveau chef du camp Nikishov pour retrouver la capacité productive des années 1930 n'eurent jamais les résultats escomptés.

6 Dans « The end of the Gulag ", Aleksei Tikhonov s'interroge sur la rapidité avec laquelle le système du travail forcé sera réformé après la mort de Stalin. L'auteur reconstruit la discussion autour d'un projet de 1951 du vice-ministre de l'Intérieur, S. S. Mamulov, sur la nécessité de réorganiser le Goulag et démontre que, depuis bien des années, la bureaucratie du MVD était consciente des problèmes qui empêchaient un fonctionnement correct des camps et de la nécessité d'une réforme radicale du système.

7 Deux chapitres sont consacrés au camp de Norilsk, construit à partir de 1935, qui deviendra l'un des complexes industriels et miniers les plus importants du Goulag. Leonid Borodkin et Simon Ertz dans "Coercion versus motivation: forced labor in Norilsk » montrent comment l'administration du Norilsklag bénéficia, tout au long de son histoire, d'une grande autonomie pour pouvoir organiser et motiver les prisonniers afin de remplir les plans qui lui avaient été confiés. On apprend dans le détail le fonctionnement des punitions et des primes - système des crédits pour la réduction de la durée des peines, introduction des salaires - et comment ces instruments de coercition et de motivation étaient utilisés, selon les différentes périodes, par les chefs de l'administration du camp.

8 "Building Norilsk », de Simon Ertz, s'interroge sur la genèse de Norilsk et sur la décision de charger le NKVD de la construction du bassin industriel autour de cette région qui aujourd'hui encore contient plus d'un tiers des réserves mondiales de nickel et $40 \%$ de celles de platine. Le cas de Norilsk est exemplaire : en quelques années, le rôle de l'OGPU d'abord, et du NKVD ensuite, va progressivement croître grâce à sa capacité à se charger 
de projets difficiles dans des conditions extrêmes. Si, dans un premier temps, l'idée avait été de confier la construction de Norilsk à différents ministères et de ne charger l'OGPU que de fournir la main-d'œuvre nécessaire, progressivement le NKVD va obtenir le monopole de la construction et de la gestion de Norilsk - conséquence également de la résistance du ministre de l'Industrie lourde G. K. Ordžonikidze à s'engager dans le projet.

Dans « The White Sea-Baltic Canal », Mikhail Morukov évoque les raisons qui motivèrent la décision de construire le canal reliant la mer Blanche à la mer Baltique et l'enjeu de sa construction pour le développement futur du Goulag, car il représenta une première démonstration d'utilisation massive de main-d'œuvre forcée dans un projet d'importance stratégique.

L'article de Christopher Joyce « The Gulag in Karelia: 1929 to 1941 » décrit la colonisation de la Carélie qui fut progressivement accomplie par des centaines de milliers de prisonniers, d'abord envoyés pour construire le canal mer Blanche-Baltique puis employés dans les camps implantés progressivement sur tout le territoire de cette république. Il démontre ensuite comment la Deuxième Guerre mondiale, avec l'évacuation de la majorité des camps établis en Carélie, modifia radicalement la place que celle-ci avait acquise dans l'histoire du Goulag. Joyce insiste sur le fait que l'expérience acquise en Carélie, notamment avec la construction du canal mer BlancheBaltique et du trust chargé de son fonctionnement, fut le point de départ de l'expansion du réseau des camps sur tout le territoire soviétique. Jusqu'à la moitié des années 1950, plusieurs figures de premier plan de l'Administration centrale des camps avaient ainsi fait une partie de leur carrière en Carélie. Ce texte met aussi en lumière l'ampleur des conflits entre autorités nationales et locales et la lutte constante que dut mener le gouvernement de la république autonome de Carélie contre l'expansion du Goulag sur son territoire en perdant progressivement le contrôle de sa politique industrielle et économique au bénéfice de l'OGPU/NKVD.

11 Ce livre a le grand mérite de ne pas se contenter de répondre aux nombreuses questions soulevées par le rôle du Goulag dans l'économie soviétique, mais aussi de comparer le travail forcé et le travail libre en mettant en relief les liens entre les deux univers. L'existence même du Goulag semble avoir finalement eu un effet «corrupteur » sur l'ensemble de l'économie soviétique en ralentissant le développement du marché du travail, des infrastructures sociales et en provoquant une dégradation des conditions de vie et de travail de l'ensemble de la main-d'œuvre du pays. 